\title{
Effect of Laparoscopic and Abdominal Hysterectomy on Urinary Incontinence
}

\author{
Gökmen Sukgen ${ }^{1}$, Ünal Türkay ${ }^{2}$
}

\begin{abstract}
Aim: Although hysterectomy is considered to be a safe operation, there are possible long-term effects of this procedure including urinary incontinence (UI) that has not been discussed much in the literature. This study investigated the effect of the hysterectomy on UI using Wilcoxon ranking and the Chi-square test of independence.

Materials and methods: Urinary incontinence symptoms of 52 patients who underwent abdominal hysterectomy (AH) and 34 patients who underwent laparoscopic hysterectomy (LH) were compared before and after the operation.

Results: The results indicated that preoperatively the participants who had LH developed higher levels of irritative symptoms, obstructive symptoms, and urogenital distress than that of participants who had AH. After the operation, Ul symptoms of the participants decreased in both of the groups, and there were no significant differences between patients who underwent $\mathrm{AH}$ and those who underwent $\mathrm{LH}$.

Conclusions: While this study provides significant information about the effects of hysterectomy on UI, the analysis of the results suggests that it is important to inform patients with evidence-based research studies about the effects of hysterectomy on UI. The findings indicated that the $\mathrm{UI}$ symptoms decreased after the hysterectomy and that there were no significant differences in UI symptoms for patients who had LH and AH. The result revealed that both of the procedures were effective in decreasing symptoms.

Clinical significance: While this study provides significant information about the effects of hysterectomy on UI, the analysis of the results suggests that it is important to inform patients with evidence-based research studies about the effects of hysterectomy on UI. Both of the procedures can be applied. However, physicians using their clinical and medical investigations and examination skills should decide which procedure is more appropriate for the patients and inform the patients about the possible outcomes of both procedures.

Keywords: Abdominal hysterectomy, Dysfunction, Incontinence, Laparoscopic hysterectomy.

Journal of South Asian Federation of Menopause Societies (2019): 10.5005/jp-journals-10032-1182
\end{abstract}

\section{INTRODUCTION}

Hysterectomy is one of the most common surgical procedures around the world. ${ }^{1,2}$ Hysterectomy is described as removal of the uterus either with or without the cervix using various surgical procedures including laparoscopy. There are two types of hysterectomy as total hysterectomy ( $\mathrm{TH}$ ) and supracervical hysterectomy $(\mathrm{SCH}){ }^{3}$ The $\mathrm{TH}$ is described as removal of both the uterine body and the cervix, while $\mathrm{SCH}$ is described as removing the uterus without the cervix. ${ }^{4}$ In comparison to $\mathrm{TH}$, the $\mathrm{SCH}$ is a more lenient surgery that has a low risk of damaging innervations of the bladder and the pelvic floor. ${ }^{3}$ Hysterectomy is conducted about $90 \%$ of the time for benign reasons including uterine fibroids, vaginal bleedings, pelvic pains, and pelvic-organ prolapses; and also most of the time it is elective. ${ }^{5}$ In any case, however, complications, including fever, hemorrhage-requiring transfusion, repair of adjacent organ injury, or life-threatening events, may occur during the operation. Although hysterectomy is considered to be a safe operation, there are possible long-term effects of this procedure including urinary incontinence (UI), which has not been discussed much in the literature. ${ }^{5}$

Urinary incontinence is described when a person involuntarily lose urine and cannot prevent its leaking out. There are various reasons including but not limited to coughing, laughing, and pregnancy. ${ }^{6}$ It is a common problem, increased by age, occurring in up to $40 \%$ of women. Bladder-control exercises and pelvic floor or Kegel exercises can help to reduce it. ${ }^{4}$

One of the long-term possible effects of hysterectomy is UI, which might be due to the operation damaging pelvic nerves and pelvic structures. Research indicated that there was no significant
${ }^{1}$ Department of Gynecology and Obstetrics, Gynecology and Obstetrics Private Clinic, Adana, Turkey

${ }^{2}$ Department of Gynecology and Obstetrics, University of Health Sciences, Derince Training and Research Hospital, Derince, Turkey

Corresponding Author: Gökmen Sukgen, Department of Gynecology and Obstetrics, Gynecology and Obstetrics Private Clinic, Adana, Turkey, Phone: +90 5334769601, e-mail: sukgeng@gmail.com

How to cite this article: Sukgen G, Türkay Ü. Effect of Laparoscopic and Abdominal Hysterectomy on Urinary Incontinence. J South Asian Feder Menopause Soc 2019;7(2):50-53.

Source of support: Nil

Conflict of interest: None

increase (if not an improvement) in incontinence 6-12 months after the hysterectomy., ${ }^{7,8}$ If the incontinence mechanism is similar to multiple birth, considering parity is a risk factor, it may not develop many years after the procedure. ${ }^{9}$ However, there was an increased risk of UI for 60 years old or older women who underwent hysterectomy. ${ }^{10,11}$ Meta-analysis investigated the effects of both $\mathrm{SCH}$ and $\mathrm{TH}$ on $\mathrm{Ul}$ and concluded no significant differences between the two procedures. Patients who had TH, however, were more likely to have UI comparing with those who had $\mathrm{SCH}^{.}{ }^{4}$

The link between hysterectomy and subsequent $\mathrm{UI}$ is not clear and there is a lack of research investigating it. However, physicians tend to experience a correlation between the hysterectomy and UI. Therefore, more research is needed to investigate the effects of hysterectomy on UI. With this regard, this study investigated the effects of hysterectomy on UI. 


\section{Materials and Methods}

\section{Participants and Procedures}

The study's ethics committee approval was obtained from Kocaeli Derince Training and Research Hospital. All the information about the procedures was given to the patients and an informed consent was taken from all of them. After obtaining an ethical approval, this study was conducted in the gynecology divisions of a private hospital located in the southern part of Turkey, from December 2014 to March 2018. The study included 52 patients who underwent abdominal hysterectomy $(\mathrm{AH})$ and 34 patients who underwent laparoscopic hysterectomy (LH). For this descriptive study, a total of 28 patients with benign indications for hysterectomy were retrieved using convenience sampling. The participants were informed about the purpose of the study, the procedures, and the complications that might occur, and asked if they would be willing to participate in the study. The participants were recruited on a volunteer basis and they were informed that not participating in the study would not have any negative effects on their treatment and relationship with their physician.

Demographic information of the volunteer participants, including age, weight, height, body mass index (BMI) and menopausal situation, use of hormone replacement treatment, and obstetric history (i.e., parity, vaginal birth count, birth interventions, birth type), was recorded. Using clinical investigation, it was determined whether the participant had urinary dysfunction problems or not. If participants had urinary dysfunction problems; the strength, type, and etiology of their problems; if those problems affect their social life; and any hygienic problems were recorded. In addition, to determine urinary symptoms and the effect of UI on the quality of life, the participants completed a symptoms survey before and after the operation.

\section{Measurements}

This study used a demographic information form and the urinary dysfunction index scale to investigate the effects of hysterectomy on UI. The urogenital distress inventory (UDI) measures the presence and frequency of stress and urge in UI symptoms. The scale is consisted of six items. The first two items measure irritative symptoms, the third and fourth questions measure stress symptoms, and the fifth and sixth questions measure obstructive symptoms. ${ }^{12}$ The participants are asked to rate the extent to which they experience the symptoms in addition to questions such as "Peeing often?" Each item is measured with a fourth-point Likert-type scale (i.e., 1-not at all, 2-a little bit, 3-moderately, 4-greatly). The raw scores are divided by item numbers and multiplied by 25 to obtain the final scores. Higher scores indicate higher levels of symptoms. Internal consistency reliability of the scale has been reported to range from 0.44 to $0.66 .^{12}$

In order to determine patients who had UI, during clinical investigations and stress-type examinations, a Q-tip test, which is a simple measurement tool to examine pelvic relaxations that may lead to stress UI, was conducted. To determine prolapse levels of the participants, the pelvic organ prolapse quantification (POP-Q) system analysis was applied to all of the participants. The POP-Q system is a site-specific system documenting the pelvic support status in women. It provides a standardized tool approved by the International Continence Society (ICS) and the American Urogynecologic Society (AUGS). The analyses mentioned above were conducted before and after the operation.

\section{Statistical Analysis}

SPSS version 11 was used to analyze the data. The variables were evaluated using the Wilcoxon sign rank test and the Chi-square test of independence, and a standard $p$ level of 0.05 was used to determine the significance. In addition, mean, frequency and standard deviation of the samples were calculated to provide descriptive information.

\section{Results}

The mean age of the participants was $54.79(S D=11.01)$, ranging from 41 years to 76 years. The results indicated that the patients who had LH were younger than patients who had $\mathrm{AH}$. The participants had a $\mathrm{BMI}$ of $30.66(\mathrm{SD}=5.29)$ on average with $\mathrm{BMI}$ ranging from 21.58 to 48.75 . In terms of $\mathrm{BMI}$, the results indicated that there was a significant difference between participants who had $\mathrm{LH}$ and $\mathrm{AH}$. Participants who had LH were lighter than participants who had $\mathrm{AH}$.

The parity of the participants ranged from 2 to 8 with a mean of 2.84. There was no significant parity differences between participants who had $\mathrm{LH}$ and $\mathrm{AH}$. The participants' number of vaginal birth ranged from 0 to 8 with a mean of 2.71. There was no significant number of vaginal birth differences between participants who had LH and AH. The patients' number of caesarian birth ranged from 0 to 2 , and $11.1 \%$ of the participants had at least one cesarean birth. There was no significant number of caesarian birth differences between participants who had $\mathrm{LH}$ and $\mathrm{AH}$. The results indicated that $69 \%$ of the participants were in the menopausal stage. Of the participants in the menopausal stage, the number of the participants who had $\mathrm{AH}$ was significantly higher than that of those who had LH. More details about the demographic characteristics and clinical information about the participants can be found in Table 1.

\section{Comparison of Urinary Dysfunction Symptoms before and after the Hysterectomy}

Detailed information regarding the comparison of urinary dysfunction symptoms before and after hysterectomy can be seen in Table 2. The results indicated that the participants who had LH displayed significantly higher preoperative levels of irritative symptoms than that of the participants who had $\mathrm{AH}$. Both groups had significant decrease in irritative symptoms after the operation. After the operation, there were no significant differences in irritative symptoms between participants who had $\mathrm{LH}$ and $\mathrm{AH}$.

The results indicated that there was no significant preoperative stress symptom differences between participants who had LH and $\mathrm{AH}$. The participants who had AH had significant decrease in stress symptoms after the operation while the participants who had LH had a slight increase in stress symptoms after the operation. After

Table 1: Demographic characteristics of the participants who underwent laparoscopic hysterectomy and the participants who underwent abdominal hysterectomy

\begin{tabular}{llll}
\hline & $\begin{array}{l}A H(n=52) \\
\text { mean } \pm S D\end{array}$ & $\begin{array}{l}L H(n=34) \\
\text { mean } \pm S D\end{array}$ & $p$ \\
\hline Age & $54.40 \pm 8.47$ & $46.00 \pm 6.65$ & 0.000 \\
BMI & $31.54 \pm 6.29$ & $29.70 \pm 3.86$ & 0.017 \\
Parity & $2.45(0-6)$ & $2.86(0-6)$ & 0.375 \\
Vaginal birth & $2.20(0-6)$ & $2.86(0-6)$ & 0.305 \\
Cesarean & $15.6 / 52(30 \%)$ & $8.5 / 34(25 \%)$ & 0.557 \\
Menopause & $39 / 52(75 \%)$ & $21.1 / 34(63 \%)$ & 0.000 \\
\hline
\end{tabular}


Hysterectomy on Urinary Incontinence

Table 2: Comparisons of the urogenital distress inventory between the participants who underwent laparoscopic hysterectomy and the participants who underwent abdominal hysterectomy

\begin{tabular}{|c|c|c|c|c|c|c|c|c|}
\hline \multirow{2}{*}{$\begin{array}{l}\text { Operation } \\
\text { type }\end{array}$} & \multicolumn{2}{|c|}{ UDI-IS scores } & \multicolumn{2}{|c|}{ UDI-SS scores } & \multicolumn{2}{|c|}{ UDI-OS scores } & \multicolumn{2}{|c|}{ UDI-6 total scores } \\
\hline & Preoperative & Postoperative & Preoperative & Postoperative & Preoperative & Postoperative & Preoperative & Postoperative \\
\hline $\mathrm{AH}$ & 31.66 & 19.16 & 19.99 & 9.99 & 14.99 & 12.49 & 22.29 & 13.6 \\
\hline LH & 52.22 & 24.44 & 19.99 & 21.1 & 48.88 & 8.88 & 40.73 & 18.51 \\
\hline$p$ & 0.002 & 0.710 & 0.133 & 0.358 & 0.012 & 0.997 & 0.002 & 0.598 \\
\hline
\end{tabular}

UDI, urogenital distress inventory; IS, irritative symptoms; SS, stress symptoms; OS, obstructive symptoms

Table 3: Comparisons of stress test positivity between the participants who underwent laparoscopic hysterectomy and the participants who underwent abdominal hysterectomy

\begin{tabular}{llll}
\hline & \multicolumn{2}{c}{ Stress test positive } \\
\cline { 2 - 3 } Groups & Preoperative & Postoperative & $p$ \\
\hline LH & $8.5 / 34(25 \%)$ & $3.4 / 34(10 \%)$ & 0.453 \\
AH & $17.1 / 52(33.30 \%)$ & $20.8 / 52(40 \%)$ & 1.000 \\
$p$ & 0.019 & 0.012 & \\
\hline
\end{tabular}

Table 4: Comparisons of Q-tip test values between the participants who underwent laparoscopic hysterectomy and the participants who underwent abdominal hysterectomy

\begin{tabular}{llll}
\hline & \multicolumn{2}{c}{ Q-tip test values (>35) } & \\
\cline { 2 - 3 } Groups & Preoperative & Postoperative & $p$ \\
\hline AH & $5.2 / 52(10 \%)$ & $2.6 / 52(5 \%)$ & 1.000 \\
LH & $4.3 / 34(13.30 \%)$ & $3.5 / 34(6.7 \%)$ & 1.000 \\
$p$ & 0.311 & 0.413 & \\
\hline
\end{tabular}

the operation, there were significant differences in stress symptoms between participants who had LH and AH. Participants who had LH had higher stress symptoms than that of participants who had $\mathrm{AH}$.

The results indicated that the participants who had LH had significantly higher level of preoperative obstructive symptoms than participants who had AH. Participants who had LH had significant decrease while participants who had $\mathrm{AH}$ had a slight decrease in obstructive symptoms after the operation. After the operation, there were no significant differences in obstructive symptoms between participants who had $\mathrm{LH}$ and $\mathrm{AH}$.

As for the total scores of UDI, the participants who had LH displayed significantly higher levels of preoperative urogenital distress than that of participants who had AH. Participants who had $\mathrm{AH}$ and $\mathrm{LH}$ had significant decrease in urogenital distress after the operation. After the operation, there were significant differences in obstructive symptoms between participants who had $\mathrm{LH}$ and $\mathrm{AH}$. Participants who had LH had higher urogenital distress than that of participants who had AH.

\section{Clinical Examinations and Diagnostic Test Results Pad Test}

The pad test results indicated that preoperatively there were four patients who had incontinence, three of them were in the participant group who had $\mathrm{AH}$ and one of them was in the group who had LH. There were no patients who had incontinence after the operation.

\section{Stress Test}

Participants who had AH had significantly higher levels of stress positivity than that of participants who had LH, both before and after the operation. While participants who had LH had a significant decrease in stress positivity after the operation, there was no significant difference in stress positivity after the operation in participants who had $\mathrm{AH}$. After the operation, participants who had LH had a significantly lower level of stress positivity comparing with the participants who had AH. Detailed information about the stress test results can be seen in Table 3.

\section{Q-tip Test}

The results indicated that although there was a decrease in ureteral mobility for both groups of the participants, there were no significant differences in urethral mobility between the participants who had $\mathrm{LH}$ and $\mathrm{AH}$ both before and after the operation. More detailed results can be seen in Table 4.

\section{Discussion}

The results indicated that before the operation the participants who had LH had higher levels of irritative and obstructive symptoms and total scores on UDI than that of the participants who had $\mathrm{AH}$. After the operation, symptoms decreased in both groups of the participants. After the operation, there were no symptomatic differences between both groups of patients, which suggests that the operation reduced symptoms for all of the participants. Although the research indicates that hysterectomy is associated with increased risk for $\mathrm{UI}$ due to possible damages in pelvic structures, ${ }^{13}$ our study found that hysterectomy had positive effects on the urogenital distress symptoms. One possible explanation for the results is that some researchers noted that problems within the female bladder, urethra or ureters might go unnoticed and those injuries are repaired at the time of the operation. Hence, it is possible that during the operation the problems within urinary system of the participants were detected and repaired.

This study used UDI to measure lower urinary tract dysfunction along with irritative, stress, and obstructive/discomfort symptoms, which would affect the health-related life quality of the participants. The results indicated that, in comparison to participants who had $\mathrm{AH}$, patients who had $\mathrm{LH}$ had a higher level of urogenital symptoms. However, it can be considered that the surgery had a more positive effect on participants who had LH as their symptoms decreased at a higher rate than that of participants who had $\mathrm{AH}$. Overall, our findings are in line with previous research indicating that hysterectomy did not have negative effects on the quality of life. ${ }^{14,15}$ Moreover, this study revealed that the hysterectomy improved the health-related life quality of patients in both groups, which is also in line with the previous research indicating that hysterectomy may positively influence physical health and social relationships. ${ }^{16}$

Other clinical examinations, including stress positivity, Q-tip test, and pad test, also indicated that both groups of the participants were experiencing the urogenital distress symptoms. After the surgery, however, the symptoms were decreased showing the positive effect of the surgery. In particular, the pad test revealed 
that four participants, who experienced UI before the operation, did not report UI after the operation.

\section{Conclusion and Clinical Significance}

The findings indicated that the UI symptoms decreased after the hysterectomy. It is important to inform patients about the results of the operation using evidence-based results. As hysterectomy is an important operation for women, and most of the patients might worry about the results of the operation, findings of this study could be used to inform women who might undergo hysterectomy. However, it is important to note that each patient is different and physicians' surgical performance might also be a factor on the operations' outcomes.

One possible explanation of why UI symptoms decreased after the hysterectomy is that it is possible that patients might go unnoticed for UI symptoms. Therefore, it is important to inform patients about the UI symptoms so that they would be aware of the symptoms and get earlier medical care.

The findings suggest that there were no significant differences in $\mathrm{UI}$ symptoms for patients who had $\mathrm{LH}$ and $\mathrm{AH}$. The result revealed that both of the procedures were effective in decreasing symptoms. Therefore, both of the procedures can be applied. However, physicians using their clinical and medical investigations and examination skills should decide which procedure is more appropriate for the patients and inform the patients about the possible outcomes of both procedures.

\section{LIMITATIONS}

There are several important limitations of this study that the readers should consider when interpreting the results. This study used two groups of patient who underwent $\mathrm{AH}$ and $\mathrm{LH}$. However, both groups had a relatively small sample size. Therefore, the findings of this study should only be generalized cautiously. Second, UI symptoms of the patients were evaluated using self-report measurement tools. It is important to consider that the results were based on the perception of the participants. Last, this study only used a six-item UI measurement assessing three types of distress symptoms. A more comprehensive measurement of urogenital distress might provide more accurate results.

\section{References}

1. Garry R. The future of hysterectomy. BJOG 2005;112(2):133-139. DOI: 10.1111/j.1471-0528.2004.00431.x.

2. Sparic R, Hudelist G, Berisava M, et al. Hysterectomy throughout history. Acta Chir lugosl 2011;58(4):9-14. DOI: 10.2298/ACI1104009S.
3. Saini J, Kuczynski E, Gretz 3rd HF, et al. Supracervical hysterectomy versus total abdominal hysterectomy: perceived effects on sexual function. BMC Womens Health 2002;2(1):1. DOI: 10.1186/14726874-2-1.

4. Robert M, Soraisham A, Sauve R. Postoperative urinary incontinence after total abdominal hysterectomy or supracervical hysterectomy: a metaanalysis. Am J Obstet Gynecol 2008;198(3):264.e1-264.e5. DOI: 10.1016/j.ajog.2007.09.033.

5. Brown JS, Sawaya G, Thom DH, et al. Hysterectomy and urinary incontinence: a systematic review. Lancet 2000;356(9229):535-539. DOI: $10.1016 /$ S0140-6736(00)02577-0.

6. Abrams $\mathrm{P}$, Andersson $\mathrm{KE}$, Birder L, et al. Fourth international consultation on incontinence recommendations of the international scientific committee: evaluation and treatment of urinary incontinence, pelvic organ prolapse, and fecal incontinence. Neurourol Urodyn 2010;29(1):213-240. DOI: 10.1002/nau.20870.

7. Kjerulff $\mathrm{KH}$, Langenberg PW, Greenaway L, et al. Urinary incontinence and hysterectomy in a large prospective cohort study in American women. J Urol 2002;167(5):2088-2092. DOI: 10.1016/S00225347(05)65091-4.

8. Thakar R. Dispelling the myth-does hysterectomy cause pelvic organ dysfunction? BJOG 2004;111(Suppl 1):20-23. DOI: 10.1111/j.14710528.2004.00461.x.

9. de Tayrac R, Chevalier N, Chauveaud-Lambling A, et al. Is vaginal hysterectomy a risk factor for urinary incontinence at long-term follow-up? Eur J Obstet Gynecol Reprod Biol 2007;130(2):258-261. DOI: 10.1016/j.ejogrb.2006.01.032.

10. Milsom I, Ekelund P, Molander U, et al. The influence of age, parity, oral contraception, hysterectomy and menopause on the prevalence of urinary incontinence in women. J Urol 1993;149(6):1459-1462. DOI: 10.1016/S0022-5347(17)36415-7.

11. Brown JS, Seeley DG, Fong J, et al. Urinary incontinence in older women: who is at risk? Study of osteoporotic fractures research group. Obstet Gynecol 1996;87(5 Pt 1):715-721. DOI: 10.1016/00297844(96)00013-0.

12. Utomo E, Korfage IJ, Wildhagen MF, et al. Validation of the urogenital distress inventory (UDI-6) and incontinence impact questionnaire (IIQ-7) in a Dutch population. Neurourol Urodyn 2015;34(1):24-31. DOI: 10.1002/nau.22496.

13. Naru T, Haq F, Rizvi JH. Urinary incontinence following gynaecological surgery. Obstet Gynaecol Reprod Med 2007;17(9):266-271. DOI: 10.1016/j.ogrm.2007.07.005.

14. Hock M, Tóth $S$, Hartmann $G$, et al. Quality of life, sexual functions and urinary incontinence after hysterectomy in hungarian women. Am J Health Res 2015;3(6):393-398. DOI: 10.11648/j.ajhr.20150306.23.

15. Thakar R, Ayers S, Georgakapolou A, et al. Hysterectomy improves quality of life and decreases psychiatric symptoms: a prospective and randomised comparison of total versus subtotal hysterectomy. BJOG 2004;111(10):1115-1120. DOI: 10.1111/j.1471-0528.2004.00242.x.

16. Lee CF, Wen FH, Lin SS, et al. Changing quality of life in hysterectomized women. J Formos Med Assoc 2009;108(5):414-422. DOI: 10.1016/ S0929-6646(09)60086-3. 